\title{
Percepção de tutores quanto à resposta clínica de cães braquicefálicos portadores de estenose de narinas submetidos a rinoplastia com o uso do laser de diodo
}

Tutors' perception of the clinical response of brachycephalic dogs with stenosis of the nostrils submitted to rhinoplasty using the diode laser

Percepción de los tutores sobre la respuesta clínica de perros braquicéfalos con estenosis de las fosas nasales sometidos a rinoplastia con láser de diodo

Recebido: 16/03/2021 | Revisado: 24/03/2021 | Aceito: 26/03/2021 | Publicado: 03/04/2021

\author{
Aguinaldo Francisco Mendes Junior \\ ORCID: https://orcid.org/0000-0002-2780-9294 \\ Universidade Federal Fluminense, Brasil \\ E-mail: aguinaldo_zootec@hotmail.com \\ Gabriela Souza Leão Bezerra dos Santos \\ ORCID: https://orcid.org/0000-0002-2454-573X \\ Universidade Federal Fluminense, Brasil \\ E-mail: gabriellaleao@id.uff.br \\ Pâmela Cristina de Souza Duarte \\ ORCID: https://orcid.org/0000-0001-7565-1519 \\ Universidade Federal Fluminense, Brasil \\ E-mail: pameladuarte2@gmail.com \\ Marina Ribeiro Hotz \\ ORCID: https://orcid.org/0000-0001-9283-8721 \\ Universidade Federal Fluminense, Brasil \\ E-mail: marinahotz@id.uff.br \\ Juliana Letícia Rosseto Marques \\ ORCID: https://orcid.org/0000-0002-2970-1594 \\ Universidade Federal Fluminense, Brasil \\ E-mail: julianamarquees.vet@gmail.com \\ Vivian Gomes Ferreira de Almeida \\ ORCID: https://orcid.org/0000-0003-1120-9427 \\ Universidade Federal Fluminense, Brasil \\ E-mail: vivian_gomes@id.uff.br \\ Gabriele Barros Mothé \\ ORCID: https://orcid.org/0000-0003-0835-5239 \\ Universidade Federal Fluminense, Brasil \\ E-mail: gabimothe2@hotmail.com \\ Ana Maria Barros Soares \\ ORCID: https://orcid.org/0000-0001-8757-1946 \\ Universidade Federal Fluminense, Brasil \\ E-mail: anasoaresvet@gmail.com \\ Nádia Regina Pereira Almosny \\ ORCID: https://orcid.org/0000-0001-7043-0717 \\ Universidade Federal Fluminense, Brasil \\ E-mail: nadiaalmosny@id.uff.br
}

\begin{abstract}
Resumo
Objetivando avaliar a resposta clínica de cães braquicefálicos portadores estenose de narinas submetidos a rinoplastia com o uso do laser de diodo, 62 tutores de cães braquicefálicos portadores de estenose de narinas foram entrevistados por meio de questionários aplicados presencialmente. Trinta e dois $(51,6 \%)$ cães eram da raça Buldogue francês, 19 $(30,6 \%)$ da raça Pug, $6(9,7 \%)$ eram Buldogues ingleses e $5(8,1 \%)$ Shih tzus. Quarenta cães $(64,5 \%)$ eram machos e $22(35,5 \%)$ fêmeas, com idade média de 29 meses. Ronco dormindo foi o sinal clínico mais relatado, 62/62 (100\%), seguido de ronco acordado, 57/62 (92\%), intolerância ao exercício, 50 (80\%), apneia do sono, 30/62 (48\%), e cianose 21/62 (34\%). Espirro reverso, espirro e tosse foram citados por $49(79 \%), 38(61 \%)$ e 27 (43\%) dos responsáveis, respectivamente. Foi constatado ainda que 82\% (51/62) dos animais apresentavam flatulência, 80\% (50/62) engasgo, $74 \%$ (46/62) vômito, $71 \%$ (44/62) regurgitação e 29\% (18/62) disfagia. Uma redução acentuada dos sinais clínicos comuns à síndrome braquicefálica foi averiguada 30 dias após rinoplastia com laser de diodo, observando-se diminuição de $15 \%$ na ocorrência de ronco dormindo, $23 \%$ de ronco acordado, $22 \%$ de intolerância ao exercício, $30 \%$ de apneia, $48 \%$ de espirro reverso, $39 \%$ de espirro, $28 \%$ de tosse e $28 \%$ de cianose. Do mesmo modo, após a cirurgia, observou-se decréscimo de $14 \%$ na ocorrência de flatulência, $41 \%$ de engasgo, $45 \%$ de vômito, $39 \%$ nos casos de
\end{abstract}


regurgitação e $19 \%$ de disfagia. O uso do laser de diodo mostrou-se eficaz na realização da rinoplastia em cães braquicefálicos portadores de estenose de narinas, promovendo redução dos sinais clínicos, com bons resultados estéticos e funcionais.

Palavras-chave: Síndrome braquicefálica; Qualidade de vida; Questionários.

\begin{abstract}
In order to evaluate the clinical response of brachycephalic dogs with stenosis of the nostrils submitted to rhinoplasty using the diode laser, 62 tutors of brachycephalic dogs with nostril stenosis were interviewed, questionnaires were applied in person. Thirtytwo (51.6\%) dogs were of the French Bulldog breed, 19 (30.6\%) of the Pug breed, 6 (9.7\%) were English Bulldogs and 5 (8.1\%) were Shih tzus. Forty dogs (64.5\%) were male and 22 (35.5\%) female, with an average age of 29 months. Sleeping snoring was the most reported clinical sign, 62/62 (100\%), followed by awake snoring, 57/62 (92\%), exercise intolerance in $50(80 \%)$, sleep apnea was reported in 30/62 (48\%), and cyanosis in 21/62 (34\%). Reverse sneezing, sneezing and coughing were cited in 49 (79\%), 38 $(61 \%)$ and $27(43 \%)$ of those responsible, respectively. The survey also found that 82\% (51/62) of the animals had flatulence, 80\% (50/62) had choking, 74\% (46/62) vomited, 71\% (44/62) regurgitation and 29\% (18/62) dysphagia. A marked reduction in clinical signs common to brachycephalic syndrome was found 30 days after performed the rhinoplasty using the diode laser, there was a $15 \%$ decrease in the occurrence of snoring while sleeping, $23 \%$ awake snoring, $22 \%$ exercise intolerance, $30 \%$ apnea, $48 \%$ reverse sneezing, $39 \%$ sneezing, $28 \%$ coughing and $28 \%$ cyanosis. Likewise, after surgery, there was a $14 \%$ decrease in the occurrence of flatulence, $41 \%$ of choking, $45 \%$ of vomiting, $39 \%$ in cases of regurgitation and $19 \%$ of dysphagia. The use of the diode laser proved to be effective in performing rhinoplasty in brachycephalic dogs with stenosis of the nostrils, promoting a reduction in clinical signs, with good aesthetic and functional results.
\end{abstract}

Keywords: Brachycephalic syndrome; Quality of life; Questionnaires.

\title{
Resumen
}

Para evaluar la respuesta clínica de de perros braquicefálicos con estenosis de las fosas nasales sometidos a rinoplastia mediante láser de diodo, se entrevistaron a 62 tutores de perros braquicefálicos con estenosis de las fosas nasales mediante cuestionarios aplicados presencialmente. Treinta y dos $(51,6 \%)$ perros eran de la raza Bulldog francés, 19 $(30,6 \%)$ de la raza Pug, $6(9,7 \%)$ eran Bulldogs ingleses y $5(8,1 \%)$ Shih tzus. Cuarenta perros $(64,5 \%)$ eran machos y $22(35,5 \%)$ hembras, con una edad media de 29 meses. Roncar mientras dormía fue el signo clínico más reportado, 62/62 (100\%), seguido de ronquidos despiertos, 57/62 (92\%), intolerancia al ejercicio, 50 (80\%), apnea del sueño, $30 / 62(48 \%)$ y cianosis 21/62 (34\%). El estornudo inverso, el estornudo y la tos fueron citados por 49 (79\%), 38 $(61 \%)$ y $27(43 \%)$ de los responsables, respectivamente. También se encontró que el 82\% (51/62) de los animales presentaba flatulencia, el 80\% (50/62) atragantamiento, el 74\% (46/62) vómitos, el 71\% (44/62) regurgitación y el $29 \%$ (18/62) 62) disfagia. 30 días después de la rinoplastia con láser de diodo se encontró una marcada reducción de los signos clínicos comunes al síndrome braquicefálico, con una disminución del $15 \%$ en la aparición de ronquidos durante el sueño, $23 \%$ de ronquidos despiertos, $22 \%$ de intolerancia al ejercicio, $30 \%$ de apnea, $48 \%$ estornudo inverso, $39 \%$ estornudo, $28 \%$ tos y $28 \%$ cianosis. Asimismo, tras la cirugía, hubo una disminución del $14 \%$ en la ocurrencia de flatulencias, $41 \%$ de asfixia, $45 \%$ de vómitos, 39\% en casos de regurgitación y $19 \%$ de disfagia. El uso del láser de diodo demostró ser efectivo en la realización de rinoplastias en perros braquicéfalos con estenosis de las fosas nasales, promoviendo una reducción de los signos clínicos, con buenos resultados estéticos y funcionales.

Palabras clave: Síndrome braquicefálico; Calidad de vida; Cuestionários.

\section{Introdução}

Inúmeras deformações obstrutivas de etiologia congênita como estenose bilateral de narinas, presença de cornetos nasais aberrantes, macroglossia, redução dos espaços naso e orofaríngeo, diminuição do espaço retrofaríngeo, prolongamento de palato mole e hipoplasia de traqueia compõem a síndrome braquicefálica. Essas alterações impactam as funções fisiológicas dos sistemas respiratório, cardiovascular e digestório, comprometendo de forma relevante o bem-estar e a qualidade de vida desses animais (Dupré \& Heidenrich, 2016).

A intensa obstrução das vias aéreas anteriores causada pelas deformações anatômicas, impedem o fluxo adequado do ar até os pulmões, aumentando o esforço inspiratório e o turbilhonamento do ar na região, e, consequentemente, suscitando um aumento poderoso da pressão negativa no interior dessas vias na cavidade torácica e abdominal, o que leva ao aparecimento de variada sintomatologia nos animais (Packer \& Tivers, 2015; Dupré \& Heidenrich, 2016; Liu et al., 2017).

Os sinais clínicos se desenvolvem em conformidade com a fisiopatologia da doença, e sua gravidade varia de acordo com o nível de obstrução das vias aéreas anteriores (Oechtering, 2010; Packer \& Tivers, 2015), podendo ter início a partir dos primeiros meses de idade, e perdurar ao longo da vida, alguns deles persistindo mesmo após o tratamento clínico e cirúrgico (Dupré e Heidenrich, 2016). 
Alterações respiratórias e gastrintestinais como dispneia, cianose, tosse, espirro, espirro reverso, ronco, intolerância ao exercício, ruídos respiratórios alterados, doenças pulmonares crônicas, apneia, disfagia, engasgo, regurgitação, flatulência, vômito, esofagite, gastrite e quadros agudos como intermação são sinais comumente relatados. (Emmerson, 2014; Packer \& Tivers, 2015; Liu et al., 2017).

Dispneia, cianose, tosse, espirro, espirro reverso, ronco, intolerância ao exercício, ruídos respiratórios alterados, doenças pulmonares crônicas, apneia, assim como, disfagia, engasgo, regurgitação, flatulência, vômito, esofagite, gastrite e quadros agudos como intermação são sinais comumente relatados. Quando expostos a exercícios físicos extenuantes e altas temperaturas, acontece a exacerbação desses sinais, aumentando o risco para esses animais (Trappler; Moore, 2011; Dupré \& Heidenrich, 2016).

Sabe-se que quanto mais anterior a resistência à entrada do ar, maior o esforço inspiratório (Koch, Arnold, Hubler \& Montavon, 2003; Meola, 2013). A estenose bilateral de narina por ser a alteração anatômica mais anterior, é um importante ponto de obstrução das vias aéreas, aumentando fortemente a sobrecarga inspiratória (Koch et al., 2003), estando presente em 50 a $100 \%$ dos casos diagnosticados exercendo impacto direto na gravidade dos sinais clínicos (Liu, Sargan, Adams \& Ladlow, 2015).

A correção cirúrgica das deformidades anatômicas que causam as diferentes obstruções mecânicas é a principal indicação para o tratamento da síndrome. Os procedimentos cirúrgicos visam diminuir a resistência e facilitar o fluxo do ar pelas vias aéreas anteriores e resultam em boa resposta clínica (Trappler \& Moore, 2011, Dupré \& Heidenrich, 2016), com mínimo risco para o paciente (Tarricone, Hayes, Singh, \& Davis, 2019).

O uso cirúrgico do laser diodo vem sendo implementado de maneira parcimoniosa para a correção de anormalidades associadas a síndrome dos braquicéfalos (Dunié-mérigot, Bouvy \& poncet, 2010; Oechtering, Pohl, Schlueter \& Schuenemann, 2016). Há inúmeras vantagens no emprego desse artifício, em comparação com os procedimentos cirúrgicos convencionais (Keats, 2012; Lodato \& Mauterer, 2014; Oechtering, Pohl, Schlueter \& Schuenemann, 2016).

O laser diodo interage com o tecido alvo e, além de realizar a ablação, tem ação bioestimuladora e sela vasos sanguíneos, linfáticos e terminações nervosas. Dessa forma, propicia melhor hemostasia, não havendo necessidade da realização de suturas, e encurta o tempo cirúrgico, com menores índices de edema, inflamação e dor no trans e no pósoperatório (Core, 2013; Lodato \& Mauterer, 2014).

Frente ao exposto, o presente estudo objetiva avaliar, por meio de questionários aplicados a tutores de cães portadores de estenose de narina submetidos a rinoplastia, a resposta clínica ao tratamento cirúrgico com o uso do laser de diodo.

\section{Metodologia}

Este estudo foi aprovado pela Comissão de Ética no Uso de Animais da Universidade Federal Fluminense (UFF), sob o número de protocolo 960/2018.

A presente pesquisa foi realizada baseada em uma abordagem quantitativa com coleta de dados numéricos por meio do uso de medições de grandezas obtidos através de metrologia e números com suas respectivas unidades, possibilitando assim a geração de conjuntos de dados passíveis de serem analisados por meio de métodos estatísticos aplicáveis ao processo (Pereira et al., 2018).

O estudo foi conduzido na Faculdade de Veterinária da Universidade Federal Fluminense (UFF), Niterói, Rio de Janeiro, Brasil, as avaliações clínicas e os procedimentos de rinoplastia, realizados no Hospital Universitário de Medicina Veterinária Professor Firmino Mársico Filho (HUVET-UFF). 
No período de junho de 2018 a junho de 2019, foram avaliados para o estudo cães de raças braquicefálicas atendidos no HUVET-UFF e encaminhados ao Projeto Narizinho UFF. Foram selecionados animais que, no exame clínico apresentassem estenose de narinas. A inspeção direta das narinas foi realizada sempre pelo mesmo médico veterinário.

Foram incluídos no estudo cães braquicefálicos, machos ou fêmeas, acima de 1 ano de idade, portadores de estenose bilateral de narinas, independentemente das demais alterações anatômicas obstrutivas comuns à síndrome braquicefálica. Todos os animais apresentavam os exames clínico e complementares isentos de alterações sistêmicas não relacionadas diretamente à síndrome dos braquicéfalos.

Foram excluídos do estudo animais abaixo de 1 ano de idade ou que apresentaram alterações respiratórias diagnosticadas no exame radiográfico, alterações cardíacas, hematológicas e/ou bioquímicas séricas hepáticas e renais que impossibilitasse submeter o animal ao procedimento cirúrgico ou anestésico. Todos os pacientes foram cadastrados em fichas individuais, numeradas em ordem crescente, contendo dados de histórico, anamnese e exame físico, além de um questionário que foi aplicado a cada tutor.

Um questionário retrospectivo adaptado de Pohl, Roedler \& Oechtering (2016) foi presencialmente aplicado ao responsável pelos animais, no momento da primeira avaliação e 30 dias após a realização da cirurgia. O questionário era constituído por questões objetivas quanto à percepção do tutor em relação à ocorrência e frequência de sinais clínicos relacionados à síndrome dos braquicéfalos. Os sinais investigados foram: tosse, espirro, espirro reverso, cianose de mucosas, intolerância ao exercício, engasgo, disfagia, regurgitação, vômito, flatulência, apneia do sono, ronco acordado e ronco dormindo.

Os sinais foram classificados em 0 , quando ausentes, 1, quando ocasionais (apresentação do sinal uma vez a cada 15 dias), 2, frequentes (apresentação do sinal de uma a mais vezes a cada sete dias) e 3, muito frequentes (apresentação do sinal de uma a mais vezes em um dia).

Os tutores responderam a dois questionários idênticos, um no momento da primeira avaliação clínica do paciente e outros 30 dias após a realização da rinoplastia, e não tiveram acesso às respostas anteriores.

O procedimento cirúrgico foi realizado sempre pelo mesmo cirurgião, utilizando aparelho DMC Vetlase® com laser diodo e comprimento de onda infravermelho $808 \mathrm{~nm} \pm 10 \mathrm{~nm}$ e potência útil de $9 \mathrm{~W} \pm 20 \%$, com fibra de diâmetro $400 \mu \mathrm{m}$ (Figura 1). Na rinoplastia foi realizada a técnica de alavestibuloplastia descrita por Mendes Junior, et al. (2021), com o laser de diodo no modo contínuo na potência de $2,8 \mathrm{~W}$, procedendo à marcação interna e externa das asas das narinas a serem retiradas (Figura 1A, B, C, D), e, em seguida, fez-se a fotoablação pela porção medial até a porção caudal da asa hipertrofiada, liberando assim a abertura da cavidade nasal (Figura 2E, F). 
Figura 1 - Realização de rinoplastia com Laser de diodo de alta potência em canino da raça Buldogue francês. Marcações das áreas a serem incisadas (A, B, C, D). Realização da fotoablação das porções externa (E) e interna (F).

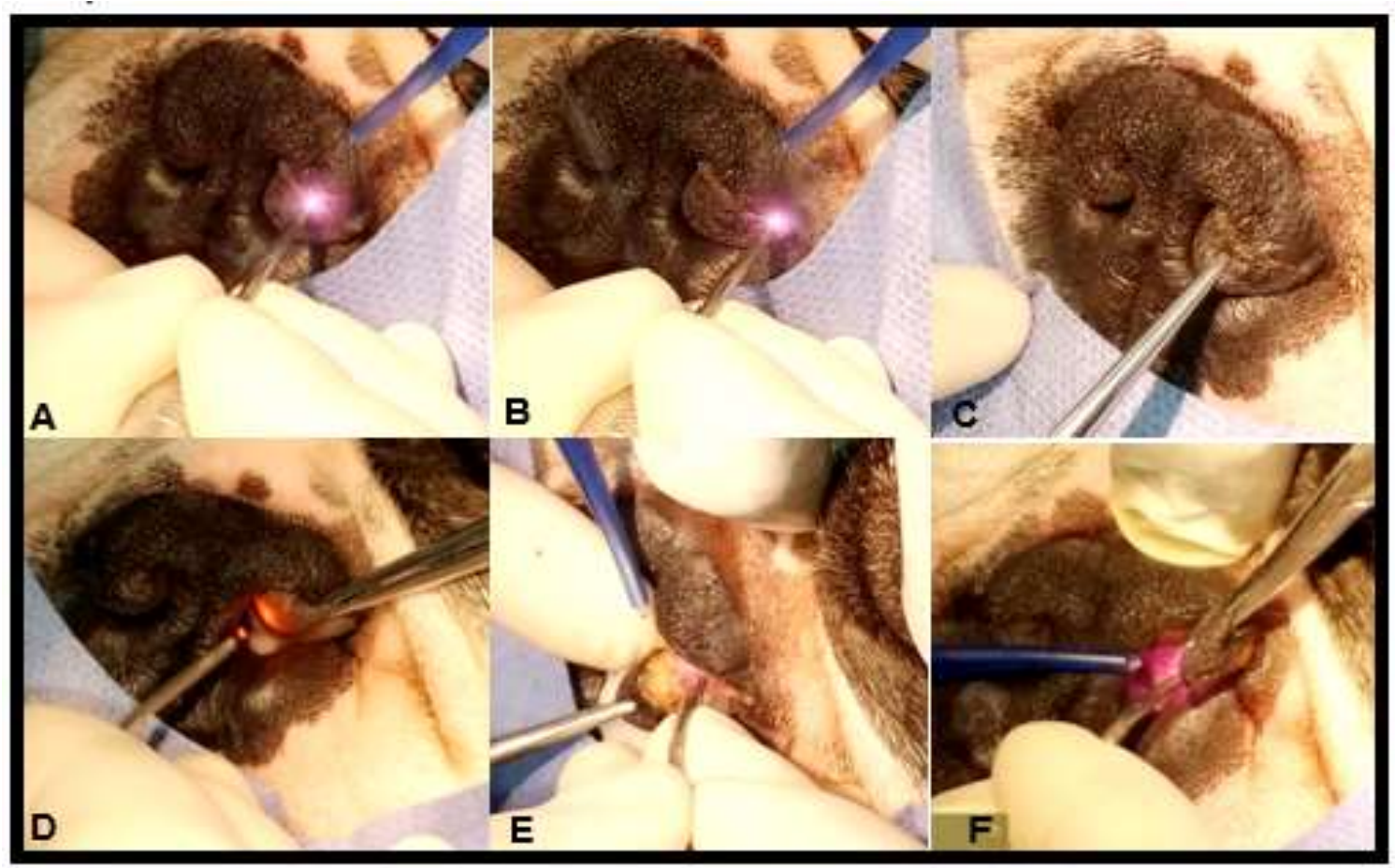

Fonte: Mendes Junior, et al. (2021).

Todos os dados coletados foram tabelados em planilhas do Microsoft Excel 2010® e submetidos a cálculo de frequência. Todas as análises estatísticas foram realizadas no programa BioEstat 5.3 (AYRES et al., 2007). Os dados foram submetidos ao teste de normalidade Shapiro-Wilk e posteriormente ao teste Qui-quadrado ou Exato de Fisher, quando necessário. Sendo significativo quando o p<0,05 (Sampaio, 2002).

\section{Resultados e Discussão}

Foram avaliados 62 cães braquicefálicos portadores de estenose de narinas; $32(51,6 \%)$ cães eram da raça Buldogue francês e 19 (30,6\%) eram da raça Pug, seguidos de seis $(9,7 \%)$ Buldogues ingleses e cinco $(8,1 \%)$ Shih tzus. No atual estudo, verificou-se que 40 cães $(64,5 \%)$ eram machos e 22 (35,5\%) fêmeas, os animais apresentavam idade média de 2,4 anos com intervalo de 1 a 9 anos e peso médio de $12 \mathrm{~kg}$, variando de 7 a $28 \mathrm{~kg}$.

Os tutores de cães braquicefálicos portadores de estenose de narinas submetidos a rinoplastia com laser de diodo responderam presencialmente a um questionário retrospectivo utilizado para coleta de dados sobre os sinais clínicos observados antes e 30 dias depois da rinoplastia. Pohl, Roedler Oechtering, (2016), realizaram avaliação similar antes e seis meses depois da realização de múltiplos procedimentos cirúrgicos corretivos das obstruções anatômicas de vias aéreas anteriores com o bisturi convencional de corte, e consideram efetivo esse método de avaliação direcionado, podendo ser mais fidedigno a estudos retrospectivos que o uso de registros médicos, sendo assim a aplicação de questionários a tutores de cães braquicefálicos pode ser útil para a avaliação da sintomatologia clínica nesses animais.

Os resultados obtidos, relativos à ocorrência e frequência dos sinais observados antes de os animais serem submetidos a rinoplastia, encontram-se dispostos na Tabela 1. 
Tabela 1 - Frequência dos sinais clínicos (categorizados como ausentes, ocasionais, frequentes ou muito frequentes) observados pelos tutores dos 62 cães braquicefálicos portadores de estenose de narinas; relatados antes de os animais serem submetidos à rinoplastia a laser.

\begin{tabular}{c|c|c|c|c|c} 
Sinais clínicos & $\begin{array}{c}\text { Ocorrência } \\
\mathrm{n}(\%)\end{array}$ & $\begin{array}{c}\text { Ausente } \\
\mathrm{n}(\%)\end{array}$ & $\begin{array}{c}\text { Ocasional } \\
\mathrm{n}(\%)\end{array}$ & $\begin{array}{c}\text { Frequente } \\
\mathrm{n}(\%)\end{array}$ & $\begin{array}{c}\text { Muito frequente } \\
\mathrm{n}(\%)\end{array}$ \\
\hline Ronco dormindo & $62(100 \%)$ & 0 & $3(5 \%)$ & $12(19 \%)$ & $47(76 \%)$ \\
\hline Ronco acordado & $57(92 \%)$ & $3(5 \%)$ & $10(17,5 \%)$ & $20(35,5 \%)$ & $27(51 \%)$ \\
\hline Flatulência & $51(82 \%)$ & $11(18 \%)$ & $12(23,5 \%)$ & $13(25,5 \%)$ & $26(51 \%)$ \\
\hline Intolerância ao exercício & $50(80 \%)$ & $12(19 \%)$ & $6(12 \%)$ & $19(38 \%)$ & $25(50 \%)$ \\
\hline Engasgo & $50(80 \%)$ & $12(19 \%)$ & $34(68 \%)$ & $10(20 \%)$ & $6(12 \%)$ \\
\hline Espirro & $49(79 \%)$ & $13(21 \%)$ & $25(51 \%)$ & $13(27 \%)$ & $11(22)$ \\
\hline Vômito & $46(74 \%)$ & $16(26 \%)$ & $30(65 \%)$ & $10(22 \%)$ & $6(13 \%)$ \\
\hline Regurgitação & $44(71 \%)$ & $18(29 \%)$ & $25(57 \%)$ & $11(25 \%)$ & $6(30 \%)$ \\
\hline Espirro reverso & $38(61 \%)$ & $24(39 \%)$ & $25(66 \%)$ & $11(29 \%)$ & $2(5 \%)$ \\
\hline Apneia do sono & $30(48 \%)$ & $32(52 \%)$ & $22(82 \%)$ & $5(18 \%)$ & 0 \\
\hline Tosse & $27(43 \%)$ & $35(56 \%)$ & $22(82 \%)$ & $5(18 \%)$ & 0 \\
\hline Cianose de mucosas & $21(34 \%)$ & $41(66 \%)$ & $14(67 \%)$ & $3(14 \%)$ & $4(19 \%)$ \\
\hline Disfagia & $18(29 \%)$ & $44(71 \%)$ & $8(45 \%)$ & $6(33 \%)$ & $4(22 \%)$
\end{tabular}

$\mathrm{n}=$ número de animais. Fonte: Autores.

O ronco foi o sinal clínico mais relatado nesse estudo. Todos os tutores afirmaram que seus cães apresentavam ronco dormindo e $76 \%$ desses animais roncavam muito frequentemente. Foi citado ainda que 27 (51\%) dos 57 cães que roncavam acordado, faziam frequentemente, validando os achados de Mendes Junior et al. (2017), que aplicaram questionário semelhante a 26 tutores de cães braquicefálicos e também constataram que 100\% dos animais roncavam dormindo e 80,7\% apresentavam ronco acordado, assim como no estudo de Gianella et al., (2019), em que 93\% dos cães roncavam e destes 56\% com muita frequência. Anteriormente, Torres e Hunt (2016) também encontraram uma alta percentagem (71\%) de cães braquicefálicos que apresentavam ronco dormindo.

Acredita-se que a alta prevalência do ronco em raças braquicefálicas se deve à acentuada diminuição da região faríngea associada ao palato mole alongado, que ocasionam aumento do esforço inspiratório e da pressão negativa, acarretando grande turbulência e vibração nessa estrutura, o que produz reverberação e, consequentemente, o ronco (Schuenemann \& Oechtering, 2014; Oechtering et al., 2016; Seneviratne, Kaye, \& Ter Haar, 2020). Outras alterações comumente identificadas nos braquicéfalos como tonsilas hipertrofiadas e macroglossia, também influenciam diretamente a ocorrência do ronco nesses animais, pois diminuem ainda mais o lúmen da orofaringe (Schuenemann \& Oechtering, 2014; Oechtering et al., 2016), deste forma sendo comum a observação deste sinal clínico em cães braquicefálicos em função do grande número de alterações anatômicas que influenciam diretamente na diminuição do lúmem da nasofaringe.

Aproximadamente metade dos cães desenvolviam episódios de apneia do sono, resultado esperado, visto que, assim como acontece com pacientes humanos portadores de obstruções em vias aéreas anteriores, os cães braquicefálicos são extremamente propensos ao desenvolvimento de distúrbios do sono, os quais são utilizados, inclusive, como modelos anatômicos para o estudo da síndrome respiratória obstrutiva do sono (Planellas, et al.,2012). Um maior relaxamento das estruturas musculares acontece no momento do sono, o que exacerba a obstrução das vias respiratórias (Planellas et al., 2015), intensificando o ronco e os quadros de apneia no período noturno. Em função disso, é comum que os cães braquicefálicos 
assumam posições não habituais ou usem objetos para manter a cavidade oral aberta durante o repouso, com o objetivo de facilitar o fluxo do ar pelas vias aéreas nesse momento (Roedler, Pohl \& Oechtering, 2013).

Espirro reverso, espirro e tosse foram sinais categoricamente citados pelos responsáveis, com uma frequência de ocorrência principalmente ocasional. Resultados menos expressivos foram vistos por Torrez \& Hunt (2006), em cujo estudo se constatou que apenas 6,3\% dos animais manifestaram espirro. Porém, os achados ora demonstrados se assemelham aos de Mendes Junior, Silva, Soares \& Almonsny, em que os autores justificam tal fato com a intensa seleção de características de braquicefalia a que esses animais são cada vez mais submetidos. Considera-se, mais uma vez, que a diminuição do espaço retrofaríngeo e a grande turbulência do ar nas vias aéreas dos braquicéfalos leve a um processo inflamatório que desencadeia um quadro agudo de irritação da mucosa e que, na inspiração ou expiração rápida e forçada do ar, produz um efeito sonoro alto característico (Pohl, Roedler \& Oechtering, 2016).

Intolerância ao exercício e cianose de mucosas foram sinais de hipóxia fortemente identificados na pesquisa ora conduzida, indo de encontro aos achados de Roedler, Pohl \& Oechtering (2013) e Gianella et al., (2019) em que 92\% e 85,7 dos tutores entrevistados, respectivamente, afirmaram que seus animais se cansavam facilmente ao praticar exercícios físicos leves. Cães braquicefálicos desenvolvem hipercapnia secundariamente a quadros de hipoventilação, levando a uma diminuição no aporte de oxigênio nos tecidos o que fundamenta os sinais clínicos apresentados devido a um aporte menor de oxigênio.

Uma alta prevalência de alterações gastrintestinais foi percebida nos cães braquicefálicos deste estudo indo de encontro aos dados levantados por Kaye, Boroffka, Haagsman, Ter Haar (2015), que aplicaram questionário a 98 tutores de cães braquicefálicos submetidos à multiplas cirurgias corretivas de obstrução em via aérea anterior e averiguaram que 56\% dos animais apresentavam regurgitação, vômito ou ptialismo. Os autores constataram também correlação entre a presença e intensidade das disfunções gastrintestinais e o grau de obstrução das vias aéreas. Gianella et al., (2019) relatam em seu estudo $76 \%$ dos cães apresentavam vômito e $60 \% \%$ regurgitação. Aventa-se que as anormalidades obstrutivas das vias aéreas anteriores impedem o fluxo adequado do ar até os pulmões, aumentando o esforço inspiratório consequentemente, suscitando um aumento poderoso da pressão negativa no interior dessas vias e na cavidade torácica e até abdominal, associado à aerofagia pela respiração oral, leva ao surgimento de alterações de trato gastrintestinal nesses animais.

Devido ao grande potencial obstrutivo da estenose das narinas e seu impacto na fisiopatogênia e sintomatologia da síndrome braquicefálica (Koch et al., 2003; Meola, 2013; Packer et al., 2015, Liu et a.,2017, Ravn-Molby et al., 2019), a única correção cirúrgica abordada neste estudo foi a rinoplastia pela técnica da alavestibulopastia, em virtude da facilidade de execução, com menores chances de complicação, além de não exigir internação do paciente.

É sabido o importante comprometimento causado pelos demais componentes da síndrome braquicefálica na sintomatologia clínica (Oechtering et al., 2016). Dessarte, cumpre ressaltar que os resultados aqui apresentados podem ser influenciados por esse fator e que não devem ser considerados como uma apologia ao uso desse único procedimento para tratamento dessa enfermidade. Esta pesquisa é uma análise da influência da correção da estenose das narinas com o uso do laser diodo na sintomatologia clínica e, consequentemente, na qualidade de vida dos animais submetidos ao tratamento nessa modalidade cirúrgica, que por ventura não possam ser submetidos aos demais procedimentos cirúrgicos corretivos.

A realização dos procedimentos que visam à desobstrução das vias aéreas é imprescindível para a diminuição dos sinais clínicos e a consequente melhora na qualidade de vida e no bem-estar dos animais (Dupré \& Heidenrich, 2016; Tarricone et al., 2019).

O tratamento cirúrgico realizado de maneira correta é essencial para a redução da sintomatologia clínica e a consequente melhora na qualidade de vida do animal ( Dupré \& Heidenrich, 2016, Ladlow; 2018). No presente estudo, o procedimento de rinoplastia com o laser de diodo permitiu a liberaçao adequada do vestíbulo nasal com excelente hemostasia e sem a necessidade de sutura (Figura 2A). Trinta dias após os cães apresentavam as narinas com aspecto estético e funcional 
considerado satisfatório (Figura 2B), reduzindo os sinais clínicos da síndrome braquicefálica em todos os animais. A comparação dos sinais clínicos observados pelos tutores antes e 30 dias depois da realização da rinoplastia encontram-se na Figura 3.

Figura 2. Aspecto das narinas de canino da raça Buldogue francês, três anos de idade, antes (A) e após a rinoplastia com Laser de diodo (B).

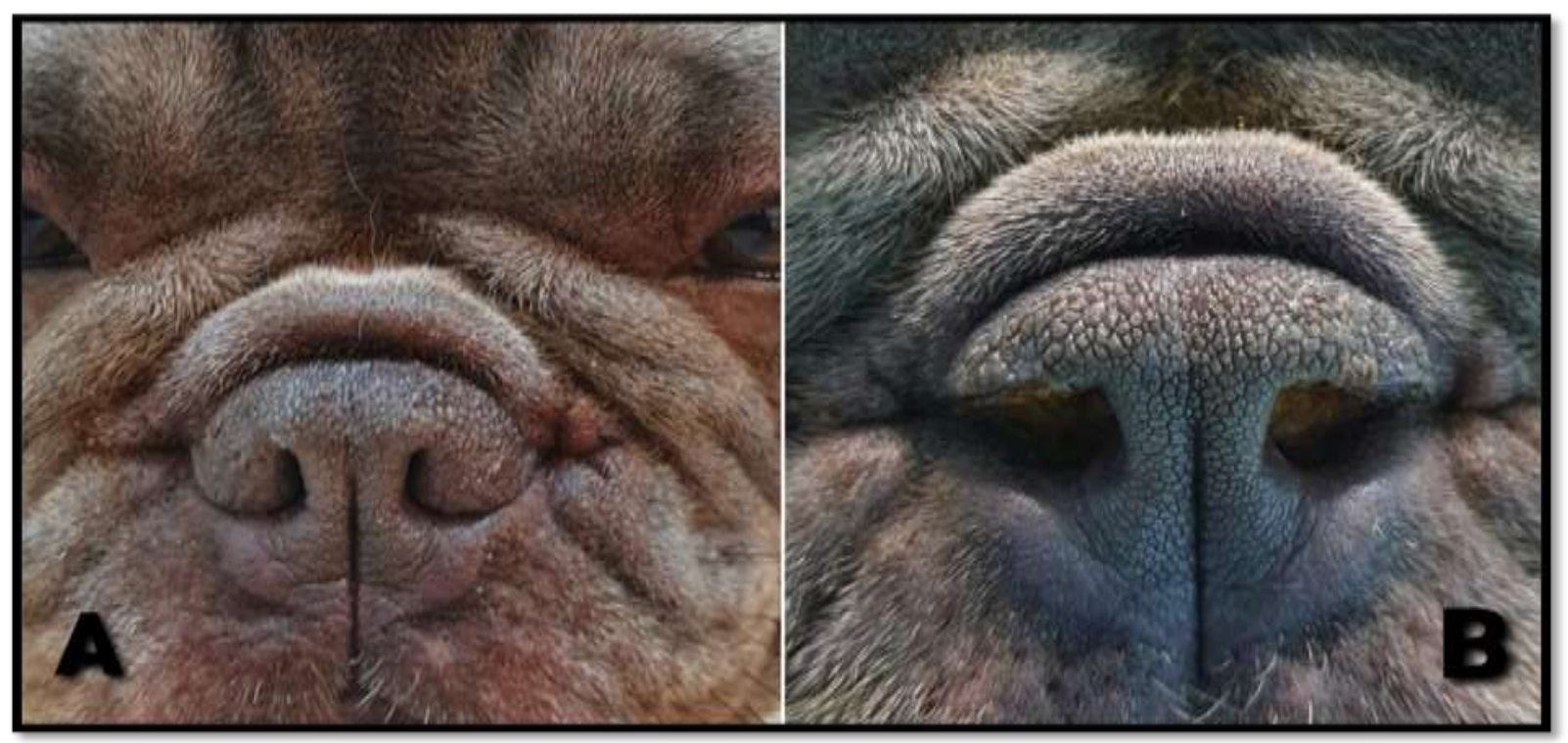

Fonte: Autores.

Figura 3 - Comparação da frequência de ocorrência dos sinais clínicos observados pelos tutores dos 62 cães braquicefálicos portadores de estenose de narinas, antes e 30 dias a serem submetidos à rinoplastia com laser de diodo.

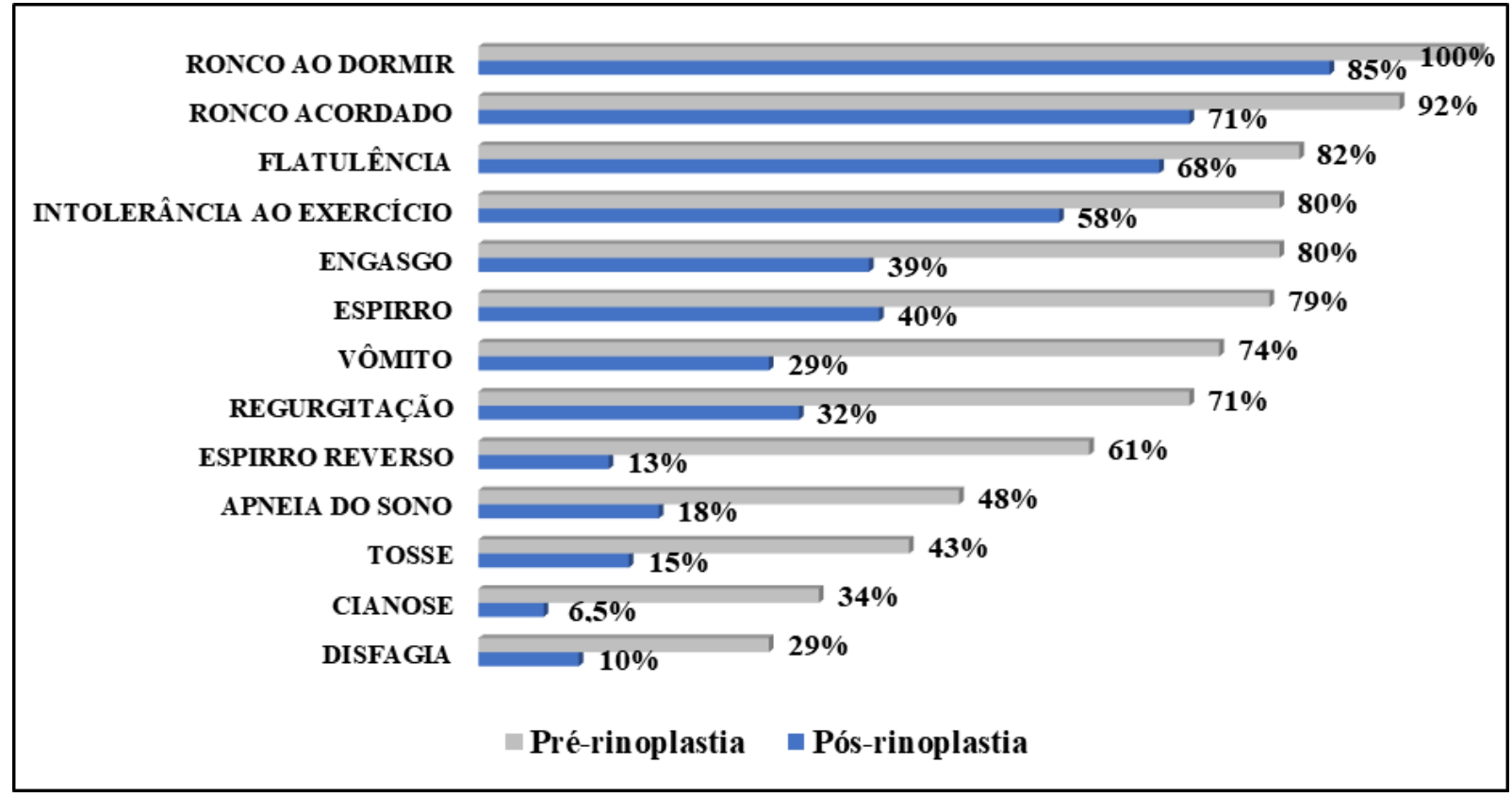

Fonte: Autores. 
Diminuição significativa foi observada nos frequentes sinais respiratórios apresentados pelos animais após a rinoplastia com laser de diodo. O percentual de redução da ocorrência dos sinais clínicos relatados pelos tutores foi calculado em função da presença ou ausência desses sinais, antes e 30 dias depois da rinoplastia. Ao aplicar o teste do Qui-quadrado, nos dados coletados nos diferentes momentos de avaliação (antes e 30 dias depois da rinoplastia), foram encontradas diferenças significativas na diminuição da ocorrência e da frequência de todos os sinais clínicos, exceto a flatulência (Tabela 2).

Tabela 2 - Comparação da frequência e redução da ocorrência dos sinais clínicos observados pelos tutores dos 62 cães braquicefálicos portadores de estenose de narinas, antes e 30 dias a serem submetidos à rinoplastia com laser de diodo.

\begin{tabular}{c|c|c|c|c}
\hline Sinais clínicos & $\begin{array}{c}\text { Pré-rinoplastia } \\
\mathrm{n}(\%)\end{array}$ & $\begin{array}{c}\text { Pós-rinoplastia } \\
\mathrm{n}(\%)\end{array}$ & $\begin{array}{c}\text { Diminuição da } \\
\text { ocorrência } \\
\mathrm{n}(\%)\end{array}$ & p-valor \\
\hline Ronco dormindo & $62(100 \%) \mathrm{a}$ & $53(85 \%) \mathrm{b}$ & $9(15 \%)$ & 0.0056 \\
\hline Ronco acordado & $57(92 \%) \mathrm{a}$ & $44(71 \%) \mathrm{b}$ & $14(23 \%)$ & 0.0056 \\
\hline Intolerância ao exercício & $50(80 \%) \mathrm{a}$ & $36(58 \%) \mathrm{b}$ & $14(22 \%)$ & 0.0113 \\
\hline Flatulência & $51(82 \%) \mathrm{a}$ & $42(68 \%) \mathrm{a}$ & $9(14 \%)$ & 0.0971 \\
\hline Engasgo & $50(80 \%) \mathrm{a}$ & $24(39 \%) \mathrm{b}$ & $26(41 \%)$ & $<0.0001$ \\
\hline Espirro & $49(79 \%) \mathrm{a}$ & $25(40 \%) \mathrm{b}$ & $24(39 \%)$ & $<0.0001$ \\
\hline Vômito & $46(74 \%) \mathrm{a}$ & $18(29 \%) \mathrm{b}$ & $28(45 \%)$ & $<0.0001$ \\
\hline Regurgitação & $44(71 \%) \mathrm{a}$ & $20(32 \%) \mathrm{b}$ & $24(39 \%)$ & $<0.0001$ \\
\hline Espirro reverso & $38(61 \%) \mathrm{a}$ & $8(13 \%) \mathrm{b}$ & $30(48 \%)$ & $<0.0001$ \\
\hline Apneia do sono & $30(48 \%) \mathrm{a}$ & $11(18 \%) \mathrm{b}$ & $19(30 \%)$ & 0.0006 \\
\hline Tosse & $27(43 \%) \mathrm{a}$ & $9(15 \%) \mathrm{b}$ & $18(28 \%)$ & 0.0008 \\
\hline Cianose & $21(34 \%) \mathrm{a}$ & $4(6.5 \%) \mathrm{b}$ & $17(28 \%)$ & 0.0003 \\
\hline Disfagia & $18(29 \%) \mathrm{a}$ & $6(10 \%) \mathrm{b}$ & $12(19 \%)$ & 0.0124 \\
\hline
\end{tabular}

Letras diferentes nas linhas indicam que há diferença estatística, pelo teste Qui-quadrado nos dados avaliados. n= número de animais. Fonte: Autores.

Trinta dias após a cirurgia, verificou-se uma redução significativa do espirro, espirro reverso, tosse, cianose e intolerância ao exercício. Decréscimo após cirurgia nesses sinais também foram citados por Pohl, Roedler e Oechtering (2016), ao avaliarem a resposta pós-cirúrgica de cães braquicefálicos seis meses após a realização dos múltiplos procedimentos cirúrgicos conduzidos em um único tempo assim como por Kaye, Boroffka, Haagsman, Ter Haar (2015), em estudo semelhante entrevistando tutores por telefone 6 semanas após os múltiplos procedimentos corretivos, esses dados são reforçados por Mendes Junior et al. (2017) ao avaliarem a resposta pós-cirúrgicas em cães braquicefálicos, 30 dias após a rinoplastia em cunha com bisturi de corte tradicional.

No atual estudo os sinais clínicos de apneia, ronco acordado e ronco dormindo também reduziram significativamente após a rinoplastia. Diferentes estudos mostraram a diminuição desses sinais em cães braquicefálicos após ressecção cirúrgica. Torrez e Hunt (2006) constataram que a realização de múltiplos procedimentos cirúrgicos provocou uma redução de $26 \%$ na ocorrência de ronco dormindo, da mesma forma que Pohl, Roedler e Oechtering (2016) constataram, após seis meses de múltiplas correções cirúrgicas, uma redução de $55 \%$ da ocorrência de ronco acordado e de $54 \%$ de ronco dormindo nos animais.

Em função da anatomia craniana característica, os cães braquicefálicos são extremamente propensos ao desenvolvimento de distúrbios do sono, muitos deles lançando mão de artifícios para manter a boca aberta durante o descanso, 
em função da grave obstrução (Planellas et al.,2012). Esse fato se deve provavelmente ao maior relaxamento muscular das estruturas nesse período, exacerbando a obstrução das vias respiratórias (Planellas et al., 2015).

Constatou-se redução significativa pós-cirúrgica nos sinais gastrintestinais de vômito, regurgitação, engasgo e disfagia. Os resultados de estudos com pacientes submetidos às múltiplas cirurgias corretivas das vias aéreas reiteram os achados desta pesquisa. Haimel; Dupré (2015) atribuem uma melhora de aproximadamente $88 \%$ nos sinais digestivos e respiratórios após a realização de rinoplastia e palatoplastia com bisturi de corte. Uma redução nos sinais digestivos de 55\% também foi descrita por Pohl, Roedler e Oechtering (2016). Mais recentemente, Mendes Junior et al. (2017) constataram uma redução de 61,9\% no engasgo e 78,6\% na disfagia em cães submetidos ao procedimento de rinoplastia.

Com base nos dados apresentados, pode-se presumir que a obstrução causada pelas alterações anatômicas de vias aéreas influência de diretamente na ocorrência e a gravidade dos sinais gastrintestinais e respiratórios nos cães braquicefálicos uma vez que esses sinais surgem e se agravam pelo aumento da pressão negativa torácica e abdominal secundária ao aumento do esforço inspiratório causado pelas obstruções de via aérea anterior (Packer et al., 2015, Liu et a., 2017). Acredita-se, desse modo, que a desobstrução cirúrgica das vias aéreas anteriores tem impacto direto na fisiopatogênia da doença, diminuindo os sinais clínicos e propiciando maior bem-estar e melhor qualidade de vida.

Uma redução mais acentuada dos sinais e sintomas comuns à síndrome braquicefálica após a rinoplastia a laser de diodo foi observada em indivíduos da raça Buldogue francês.

Acredita-se que o maior número de indivíduos desta raça apresentados ao estudo pode ter influenciado neste resultado. Uma maior prevalência de Buldogues franceses também foi averiguada por Mendes Junior et al., 2019, que desenvolveram, na mesma região do atual estudo, avaliação comparativa dos valores de hemogasometria antes e depois da realização de rinoplastia em cães braquicefálicos e concluíram que há maior predileção dos tutores do estado do Rio de Janeiro pela raça Buldogue francês.

No presente estudo, constatou-se também uma redução significativa em um maior número de sinais clínicos nos machos (Tabela 3), e em cães de peso corporal entre 10 e 20kg. Esse fato pode estar associado a tendência de cães machos a apresentarem maior volume de massa muscular corporal, inclusive em região de face e crânio, levando a maior compressão das vias aéreas que estão envolvidas por tecidos moles (Oechtering, 2010). Também já foi confirmado que a obesidade é um fator de risco para a síndrome braquicefálica, pois provoca deposição de tecido adiposo, causando um estreitamento da faringe e maior colapsabilidade das vias aéreas superiores (Mamens, 2012). Portanto, manter cães braquicefálicos com escore de condição corporal adequado é essencial para um bom prognóstico do paciente. 
Tabela 3 - Percentual de redução e comparação da frequência dos sinais clínicos por sexo nos 62 cães braquicefálicos portadores de estenose de narinas 30 dias após serem submetidos a rinoplastia.

\begin{tabular}{c|c|c|c|c}
\hline Sinais clínicos & Macho & Fêmea & $\begin{array}{c}\text { Redução da ocorrência } \\
\mathbf{n}(\%)\end{array}$ & p-valor \\
\hline Ronco dormindo & $6(66.7 \%) \mathrm{a}$ & $3(33.3 \%) \mathrm{a}$ & $9(15 \%)$ & 0.3469 \\
\hline Ronco acordado & $9(64.3 \%) \mathrm{a}$ & $5(35.7 \%) \mathrm{a}$ & $14(23 \%)$ & 0.2568 \\
\hline Intolerância ao exercício & $10(71.4 \%) \mathrm{a}$ & $4(28.6 \%) \mathrm{a}$ & $14(22 \%)$ & 0.0570 \\
\hline Flatulência & $8(88.9 \%) \mathrm{a}$ & $1(11.1 \%) \mathrm{b}$ & $9(14 \%)$ & 0.0034 \\
\hline Engasgo & $19(73.1 \%) \mathrm{a}$ & $7(26.9 \%) \mathrm{b}$ & $26(41 \%)$ & 0.0011 \\
\hline Espirro & $15(62.5 \%) \mathrm{a}$ & $9(37.5 \%) \mathrm{a}$ & $24(39 \%)$ & 0.1482 \\
\hline Vômito & $19(70.4 \%) \mathrm{a}$ & $8(29.6 \%) \mathrm{b}$ & $28(45 \%)$ & 0.0060 \\
\hline Regurgitação & $17(70.8 \%) \mathrm{a}$ & $7(29.2 \%) \mathrm{b}$ & $24(39 \%)$ & 0.0087 \\
\hline Espirro reverso & $27(62.9 \%) \mathrm{a}$ & $13(37.1 \%) \mathrm{b}$ & $30(48 \%)$ & 0.0356 \\
\hline Apneia do sono & $16(80 \%) \mathrm{a}$ & $3(20 \%) \mathrm{b}$ & $19(30 \%)$ & 0.0004 \\
\hline Tosse & $12(63.2 \%) \mathrm{a}$ & $7(36.8 \%) \mathrm{a}$ & $18(28 \%)$ & 0.1220 \\
\hline Cianose & $13(76.5 \%) \mathrm{a}$ & $4(23.5 \%) \mathrm{b}$ & $17(28 \%)$ & 0.0028 \\
\hline Disfagia & $7(58.3 \%) \mathrm{a}$ & $5(41.7 \%) \mathrm{a}$ & $12(19 \%)$ & 0.6843 \\
\hline
\end{tabular}

Letras diferentes nas linhas indicam que há diferença estatística, pelo teste Exato de Fisher, nos dados avaliados. Fonte: Autores.

Mediante a avaliação da idade dos pacientes deste estudo e da redução dos sinais clínicos, constatou-se que animais com até três anos de idade mostram uma redução mais expressiva dos sinais clínicos, a saber, cianose, 9/17(53\%) (p < 0.0001); espirro reverso, 20/30(66,7\%) ( $\mathrm{p}=0.0003)$; espirro, 14/24(58\%) ( $\mathrm{p}=0.0003)$ flatulência, 7/9 (78\%) (p<0.0001); intolerância ao exercício, 9/14(64\%) ( $\mathrm{p}=0.0046)$; regurgitação, 15/24(62,5\%) ( $\mathrm{p}=0.0003)$; tosse, 11/18(61\%) ( $<<0.0001)$; e ronco acordado, 10/14 (71,5\%) $(\mathrm{p}<0.0001)$. Os achados corroboram os do estudo de Dupré e Heidenrich (2016) em que os autores apontam o caráter progressivo da doença e salientam melhores resultados pós-cirúrgicos em animais mais jovens, uma vez que esses animais apresentam menos alterações obstrutivas secundárias. A correção cirúrgica deve ser realizada de maneira precoce, com o objetivo de minimizar a progressão da doença, limitando o aparecimento de alterações secundárias que podem ocorrer pelo aumento da pressão negativa e pela turbulência do ar secundários à obstrução (Trappler \& Moore, 2011, Lodato \& Hedlund, 2012), desta maneira entende-se que pacientes mais jovens alcançam melhor resultados pós-cirúrgicos quando comparados aos de idade mais avançada.

Por esse motivo, em função do potencial de progressão da doença com o avançar da idade dos animais, que leva ao surgimento de alterações secundárias e agrava o estado clínico do paciente, sugere-se a realização precoce das correções cirúrgicas dos componentes da síndrome, com vistas a um melhor prognóstico na vida adulta (Trappler; Moore, 2011).

Outro ponto a ser considerado é que os responsáveis por cães braquicefálicos mostram-se mais tolerantes aos sinais de obstrução de via aérea apresentados pelos animais, e só buscam o médico veterinário quando há um nível avançado de comprometimento respiratório (Packer et al., 2012), o que pode diminuir a resposta clínica em animais mais idosos.

Cumpre ressaltar que a melhora clínica dos pacientes em resposta ao tratamento cirúrgico deve ser avaliada de forma individual, ponderando-se aspectos como a idade, as alterações anatômicas obstrutivas e a condição geral do animal.

\section{Conclusão}

O uso do laser de diodo mostrou-se eficaz na realização da ablação das asas das narinas hipertrofiadas em cães portadores da síndrome braquicefálica, promovendo redução dos sinais clínicos 30 dias após a rinoplastia, com bons resultados estéticos e funcionais. 
Apesar da grande influência das demais anomalias obstrutivas da síndrome braquicefálica na gravidade da doença, os cães acometidos se beneficiam da rinoplastia com o laser de diodo, com importante melhora da qualidade de vida e bem estar do animal.

Sugere-se por fim estudos futuros visando avaliar a redução dos sinais clínicos após a realização de múltiplos procedimentos corretivos dos componentes obstrutivos da síndrome braquicefálica.

\section{Referências}

Ayres, M., Ayres, M. J., Ayres, D. L. \& Santos, A. S. (2007). Bioestat 5.0. Aplicações estatísticas nas áreas das ciências biomédicas. Mamiraua, Belém, PA. 364.

Core, B. D. M. (2013). CO2 laser allows bloodless repair of stenotic nares. Aesculi Aesculightght, 1-2,

Dupré, G. \& Heidenreich, D. (2016.) Brachycephalic Syndrome. Journal of Small Animal Practice, 46, 4, $691-707$.

Emmerson, T. (2014). Brachycephalic obstructive airway syndrome: A growing problem. Journal of Small Animal Practice, 55(11), 543-544.

Gianella, P., Caccamo, R., Bellino, C., Bottero, E., Fietta, F., Roncone, S., Ostanello, F. Pietra, M., \& Buracco, P. (2019) Evaluation of metabolic profile and C-reactive protein concentrations in brachycephalic dogs with upper airway obstructive syndrome. Journal of Veterinary Internal Medicine, $33,2183-2192$.

Oechtering, G. U. (2010). Síndrome braquicefálica - novas informações sobre uma antiga doença congénita. Veterinary Focus, 20 (2), $2-8$.

Packer, R. M. A. \& Tivers, M. S. (2015). Strategies for the management and prevention of conformation-related respiratory disorders in brachycephalic dogs. Veterinary Medicine: Research and Reports, 6, 219-232.

Trappler, M. \& Moore, K. (2015). Canine brachycephalic airway syndrome: surgical management. Compendium: continuing education for veterinarians, 33(5), E1-E8.

Kaye, B. M., Boroffka, S. A., Haagsman, A. N, \& Ter Haar, G. (2015). Computed tomo- graphic, radiographic, and endoscopic tracheal dimensions in English Bulldogs with grade 1 clinical signs of brachycephalic airway syn- drome. Veterinary Radiologic Ultrasound. 56, 609-616.

Keats, M. M. (2012). Brachycephalic airway syndrome, part 1: correcting stenotic nares. DVM New Magazine, p.6S-8S.

Koch, D. A., Arnold, S., Hubler, M. \& Montavon, P. M. (2003). Brachycephalic syndrome in dogs. Compendium on Continuing Education for the Practicing Veterinarian, 25(1), 48-55.

Ladlow, J., Liu, N. C., Kalmar, L. \& Sargan, D., (2018). Brachycephalic obstructive airway syndrome. Veterinary Record, 182, $13,75-78,2018$.

Liu, N. C., Sargan, D. R., Adams, V. J. \& Ladlow, J. F. (2015). Characterisation of Brachycephalic Obstructive Airway Syndrome in French Bulldogs Using Whole-Body Barometric Plethysmography. PLOS ONE, 10(6), e0130741.

Liu, N. C., Troconis, E. L., Kalmar, L., Price, D. J. Wright, H. E. Adams, Vicki J. Sargan, D. R. \& Ladlow, J. (2017). Veterinary Surgery, 46, 2, 271-280.

Lodato, D., \& Mauterer, J. V. (2014). Techniques for performing corrective surgery: dogs with brachycephalic airway syndrome. Today's Veterinary Practice, 34, 8 .

Manens, J. (2014). Effect of body weight loss on cardiopulmonary function assessed by 6-minute walk test and arterial blood gas analysis in obese dogs. Journal of Veterinary Internal Medicine, 28, 2, 371-378.

Mendes Junior, A. F., Silva, G. S. D., Soares, A. M. B. \& Almonsny, N. R. P. (2017). Percepção de tutores quanto aos sinais clínicos em cães braquicefálicos portadores de estenose de narina. Enciclopédia Biosfera, Centro Científico Conhecer. 25, 530-543.

Mendes Junior, A. F., Hotz, M. R., Duarte, P. C. de S., Santos, G. S. L. B., Sanches Neto, A. P., Mothé, G. B., Soares, A. M. B. \& Almosny, N. R. P. (2021). Use of laser diode for surgical correction of stenosis of nostrils and elongated soft palate in dog with brachycephalic syndrome - case report. Research, Society and Development, 10, 2, e33410212630.

Oechtering, T. H., Oechtering, G. U. \& Nöller, C. (2007). Structural characteristics of the nose in brachycephalic dog breeds analysed by computed tomography. Tieraerztl Prax, 35, 177-187.

Oechtering, G. U., Pohl, S., Schlueter, C. \& Schuenemann, R. (2016). A Novel Approach to Brachycephalic Syndrome. Veterinary Surgery, 45, 2 , 165-172.

Pereira, A. S., Shitsuka, D. M., Pereira, F. J., Shitsuka, R. (2018). Metodologia da pesquisa científica. UFSM.

Planellas, M., Cuenca, R., Tabar, M. D. Bertolani, C. Poncet, C. Closa, J. M., Lorente, J., Cerón, J. J. \& Pastor, J. (2012). Evaluation of C-reactive protein, haptoglobin and cardiac troponin 1 levels in brachycephalic dogs with upper airway obstructive syndrome. BMC Veterinary Research, 8, 152.

Planellas, M., Cuenca, R., Tabar, M. D., Bertolani, C., Poncet, C., Closa, J.M. Lorente, J., Cerón, J. J. \& Pastor, J. (2015). Clinical assessment and C-reactive protein (CRP), haptoglobin (Hp), and cardiac troponin i (cTnI) values of brachycephalic dogs with upper airway obstruction before and after surgery. Canadian Journal of Veterinary Research, 79, 1, 58-63. 
Research, Society and Development, v. 10, n. 4, e14210413941, 2021

(CC BY 4.0) | ISSN 2525-3409 | DOI: http://dx.doi.org/10.33448/rsd-v10i4.13941

Pohl, S., Roedler, F. S. \& Oechtering, G. U. (2016). How does multilevel upper airway surgery influence the lives of dogs with severe brachycephaly? Results of a structured pre- and postoperative owner questionnaire. Veterinary Journal, 210, 39-45.

Ravn-Molby, E. M. Sindahl, Nielsen, S.S. Brunn, C. S. Sandoe, P. \& Fredholm, M. (2019). Breeding French bulldogs so that they breathe well-A long way to go. PLos One, 14,1-14.

Roedler, F. S., Pohl, S. \& Oechtering, G. U. (2013) How does severe brachycephaly affect dog's lives? Results of a structured preoperative owner questionnaire. Veterinary journal, 198, 3, 606-610.

Sampaio, I. B. M. (2002). Análise de Variância. Estatística Aplicada à Experimentação Animal. 2.ed. Fundação de Estudo e Pesquisa em Medicina Veterinária e Zootecnia, Belo Horizonte, 43-47.

Schuenemann, R. \& Oechtering, G. (2014). Inside the brachycephalic nose: Conchal regrowth and mucosal contact points after laser-assisted turbinectomy. Journal of the American Animal Hospital Association, 50, 4.

Seneviratne, M., Kaye, B. M. \& Ter Haar, G. (2020). Prognostic indicators of short-term outcome in dogs undergoing surgery for brachycephalic obstructive airway syndrome. Veterinary Record, 187(10), 403-403.

Tarricone, J., Hayes, G. M., Singh, A. \& Davis, G. (2019). Development and validation of a brachycephalic risk (Brisk) score to predict the risk of complications in dogs presenting for surgical treatment of brachycephalic obstructive airway syndrome. Veterinary Surgery: 48, 7, $1253-1261$. 